\title{
Effect of elevated intraocular pressure on the thickness changes of cat laminar and prelaminar tissue using optical coherence tomography ${ }^{1}$
}

\author{
Qiuyun Zhao ${ }^{\mathrm{a}, \mathrm{b}}$, Xiuqing Qian ${ }^{\mathrm{a}, \mathrm{b}}$, Lin Li ${ }^{\mathrm{a}, \mathrm{b}}$, Weijian Sun ${ }^{\mathrm{a}, \mathrm{b}}$, Shan Huang ${ }^{\mathrm{a}, \mathrm{b}}$ and Zhicheng \\ $\mathrm{Liu}^{\mathrm{a}, \mathrm{b}, *}$ \\ ${ }^{a}$ School of Biomedical Engineering, Capital Medical University, Beijing 100069, China \\ ${ }^{b}$ Beijing Key Laboratory of Fundamental Research on Biomechanics in Clinical Application, Capital \\ Medical University, Beijing 100069, China
}

\begin{abstract}
The aim of this study was to examine shape the changes of the lamina cribrosa (LC) under different intraocular pressures (IOPs) with different periods. Images of the optic nerve head were obtained using enhanced depth imaging spectral domain optical coherence tomography (EDI SD-OCT). After an initial scan of the IOP at native pressure, subsequent scanning was taken when the IOP values reached 40,60, 80 and $100 \mathrm{~mm} \mathrm{Hg}$. Then scans continued with the IOP maintained at $100 \mathrm{~mm} \mathrm{Hg}$ for 1 hour, 2 hours, 3 hours and 4 hours. The thicknesses of the LC and prelaminar tissue were measured and the curvature of the LC was calculated. Our study found that as IOP increased, the thicknesses of both LC and prelaminar tissue decreased and the thickness variation of the LC correlated significantly with the increases of IOP when IOP was higher than $60 \mathrm{~mm} \mathrm{Hg}$. An exponential function was proposed to express the relationship between IOP and the thickness variations of LC and prelaminar tissue. Creep curves of the LC and prelaminar tissue was also obtained using the Prony model. In conclusion, both the thickness of the prelaminar tissue and LC thinned as the IOP elevated. The thickness of the LC also decreased after 4 hours of constant $100 \mathrm{~mm} \mathrm{Hg}$ pressure.
\end{abstract}

Keywords: Laminar cribrosa, prelaminar tissue, curvature, high intraocular pressure, EDI-OCT

\section{Introduction}

As one of the significant leading cause of blindness, glaucoma comprises a group of retrogressive optic neuropathies with retinal ganglion cell apoptosis, optic nerve head (ONH) damage and a concomitant pattern of visual field loss [1-4]. Even though the pathogenesis of glaucoma is uncertain, pathological elevated IOP is thought to be the main risk. There are accumulating evidences that the laminar cribrosa (LC) region is the principal site of retinal ganglion cell (RGC) axonal insult in glau-

\footnotetext{
${ }^{1}$ Qiuyun Zhao and Xiuqing Qian contributed equally to this work.

${ }^{*}$ Corresponding author: Zhicheng Liu, School of Biomedical Engineering, Capital Medical University, Beijing 100069, China. Tel.: 086-010-83911559; Fax: 086-010-83911560; E-mail: zcliu@ccmu.edu.cn.
}

0959-2989/14/\$27.50 @ 2014 - IOS Press and the authors. 
coma [5-9]. Many investigators have implicated the role of shape changes of the LC in the pathogenesis of glaucomatous optic neuropathy. Therefore, it is important to study the relationship between shape changes of the LC and high IOP.

$\mathrm{LC}$ is the porous connective tissue of the $\mathrm{ONH}$ through which retinal ganglion cell axon bundles pass. By examining human ONHs using scanning electron microscopy, Quigley and Addicks [10] found the regional differences in the fine structure of the LC. Then, Quigley and colleagues [11] found that compression of the successive LC sheets was the earliest abnormality in glaucoma in some cases before visual field loss was detected, and backward bowing of the entire LC was a later change. The histological results [12] showed that LC thickness and peripapillary sclera thickness decreased significantly with increasing axial length, along with the thinning of the LC caused by glaucoma. Quigley and Addicks studied monkey eyes and revealed that chronic ocular hypertension would result in changes in the LC, including outward displacement of the posterior lamina [13]. However, the process of histological examination requires sectioning of the $\mathrm{ONH}$ [14], which is destructive and may cause tissue deformation.

Kagemann and colleagues reported the development of non-invasively in vivo LC imaging with spectral domain optical coherence tomography (SD-OCT) [15]. In 2009, the appearance of the ONH and LC in glaucomatous patients was evaluated. The 3D structure of the LC was resolved and its thickness was first measured with high-speed SD-OCT [16]. They found that the thickness of the LC significantly correlated with visual field loss in living patients with glaucoma.

With the development of OCT, enhanced depth imaging optical coherence tomography (EDI-OCT) was gradually and extensively applied in study of the LC. In 2011, Lee and colleagues found the fullthickness of the LC could be visualized using EDI SD-OCT and images were clearer than those obtained with previous OCT technology [17]. Recent reports have suggested that EDI-OCT could visualize the posterior border of the LC $[18,19]$, measure the full thickness of the choroid and investigate the LC [20-25]. They studied the displacement and thickness of the LC and changes that occurred after trabeculectomy and intraocular pressure reduction.

In a recent study, Park HY and Park CK studied 144 patients with glaucoma (68 with primary openangle glaucoma [POAG], the others with normal-tension glaucoma [NTG]) and 65 healthy controls. Their results showed that changes in the LC thickness were related to diagnose of glaucoma. They suggested that the diagnostic ability was comparable to that of assessing the of peripapillary retinal nerve fiber layer (RNFL) thickness and that it was even better than the later methods for patients with early NTG [26]. They concluded that the investigation of the LC facilitated the study of the pathogenesis of glaucoma. The relationship between LC thickness and changes in IOP was not considered in their studies.

Fatehee et al. [27] investigated the effects of acute elevations in IOP on cup, prelaminar and LC regions of the porcine ONH. They found that the thickness of the LC area and prelaminar tissue area both decreased. Strouthidis and colleagues studied monkey eyes and showed acute IOP elevation resulted in a reduction of the prelaminar tissue thickness [28].

The SD-OCT (3D OCT-1000 mark II , Topcon Corp, Tokyo, Japan) in lab has a special module that functions as the EDI OCT. Using this device, we could obtain sharper images of the LC. In this study, we analyzed changes in the LC under different IOPs, and also evaluated changes in the LC after different time intervals under constant $100 \mathrm{~mm} \mathrm{Hg}$ intraocular pressure. 


\section{Methods}

\subsection{Study subjects}

Since the anatomy and development of the ONH in cats are similar to those in humans [29], we chose cats as the experimental animal for this research. All the cats were provided by the Experimental Animal Department of Capital Medical University. All subjects were healthy with no ophthalmic diseases.

Five cats were used to study the in-time changes of the LC while IOP was elevated and the effect of constant $100 \mathrm{~mm} \mathrm{Hg}$ pressure on the LC. They were treated and managed in agreement with the National Institutes of Health Guide for the Care and Use of Laboratory Animals and approved by the Institutional Animal Care and Use Committee of China. Sedation was induced by an intraperitoneal injection of $25 \%$ urethane.

\subsection{High intraocular pressure model}

One 24-gauge micro-cannula was inserted into the anterior chamber of a cat and the cannula was connected to a pressure transducer. An adjustable-height infusion of normal saline was administered through a triple valve so that IOP could be monitored and continually adjusted (Figure 1).

The right eye was used as the control eye with no conduction, and whereas the left eye was used as the high-IOP model. In this paper, we employed two high-IOP models. In one model, the IOP rose to $40 \mathrm{~mm} \mathrm{Hg}, 60 \mathrm{~mm} \mathrm{Hg}, 80 \mathrm{~mm} \mathrm{Hg}$ and $100 \mathrm{~mm} \mathrm{Hg}$ successively, and in the other, the IOP was devised to be maintained at $100 \mathrm{~mm} \mathrm{Hg}$ for 4 hours. In the first model, a syringe filled with normal saline was put on the micro-syringe pump so that the IOP could be rapidly elevated as the solution was pumped into the anterior segment. The pressure transducer and the monitor system were used to supervise the IOP in real time. Four scans were completed within half an hour to reduce the effect of creep. In the second model, the physiological saline was used to adjust and maintain the IOP when it rose to the target pressure. Four scans were performed with the IOP maintained at $100 \mathrm{~mm} \mathrm{Hg}$ for 1 hour, 2 hours, 3 hours and 4 hours respectively to study the viscoelasticity of the LC and prelaminar tissue.

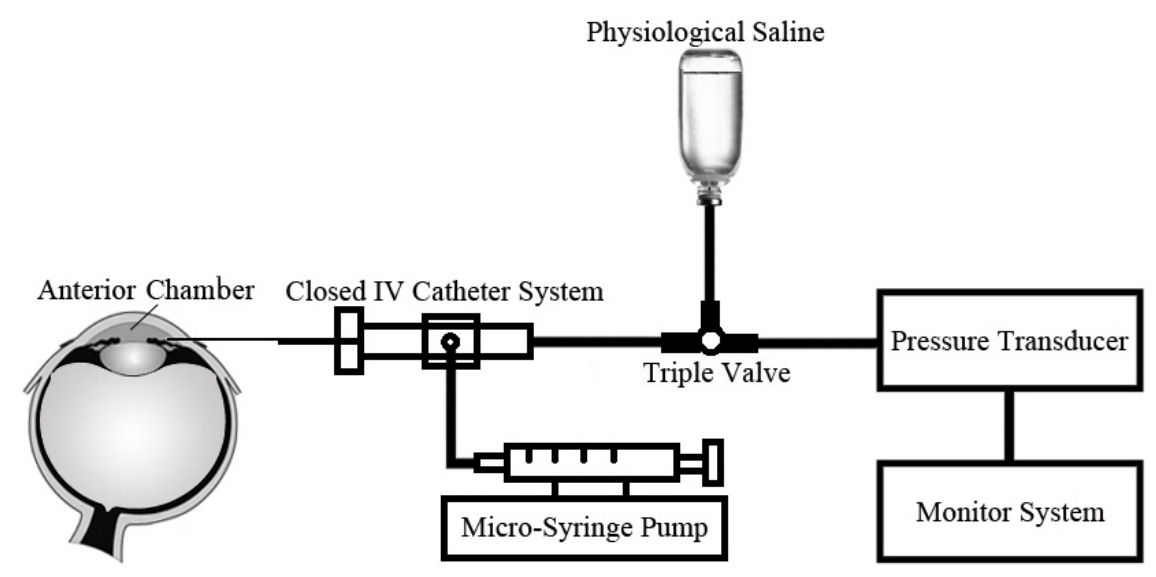

Fig. 1. Diagram of the experimental platform used for $\mathrm{ONH}$ images acquirement in our study. 


\subsection{Imaging of the prelaminar tissue and LC}

The LC was imaged with high speed SD-OCT (3D OCT-1000 mark II, Topcon Corp, Tokyo, Japan) using the choroidal mode, which can function equivalently to the enhanced depth-imaging technique.

The images were acquired by a continuous parallel scan function focusing on a rectangular region near the ONH. The parameter sets were similar to our earlier report [30]. The area volume was $6.0 \times$ $6.0 \times 2.3 \mathrm{~mm}^{3}$ (horizontal $\times$ vertical $\times$ axial) with a matrix size of $512 \times 128 \times 650$ pixels, respectively.

For comparison, we first captured one scan before perfusion, which represented the physiological condition. Then scans were acquired immediately when the IOP values reached $40 \mathrm{~mm} \mathrm{Hg}, 60 \mathrm{~mm} \mathrm{Hg}$, $80 \mathrm{~mm} \mathrm{Hg}$ and $100 \mathrm{~mm} \mathrm{Hg}$. Finally, four scans were obtained with the IOP maintained at $100 \mathrm{~mm} \mathrm{Hg}$ for 1 hour, 2 hours, 3 hours and 4 hours, respectively.

\subsection{Measuring the thicknesses of LC and prelaminar tissue}

The LC appears as a region of high back reflection underneath the bottom of the optic disc cup in OCT [9]. The thickness is defined as the distance between the anterior border (line $\beta$ ) and posterior border (line $\gamma$ ) of the LC and the posterior borders of the highly reflective region of the ONH. The prelaminar tissue thickness is defined as the distance between the optic cup surface (line $\alpha$ ) and the anterior border of the LC (line $\beta$ ) (Figure 2) [17].

$128 \mathrm{~B}$-scan images were obtained and approximately 15 pieces covered the $\mathrm{ONH}$. The middle three images were selected for thickness measurements. Before being measured, the images were optimized with image processing software (MATLAB R2009a, MathWorks, Natick, USA). Here morphological operation was applied in the program [31]. The optimization program flowchart and partial results are displayed in Figures 3 and 4. Additional software (ImageJ, National Institutes of Health, Bethesda, USA) was also used to measure the thicknesses of the prelaminar tissue and LC.

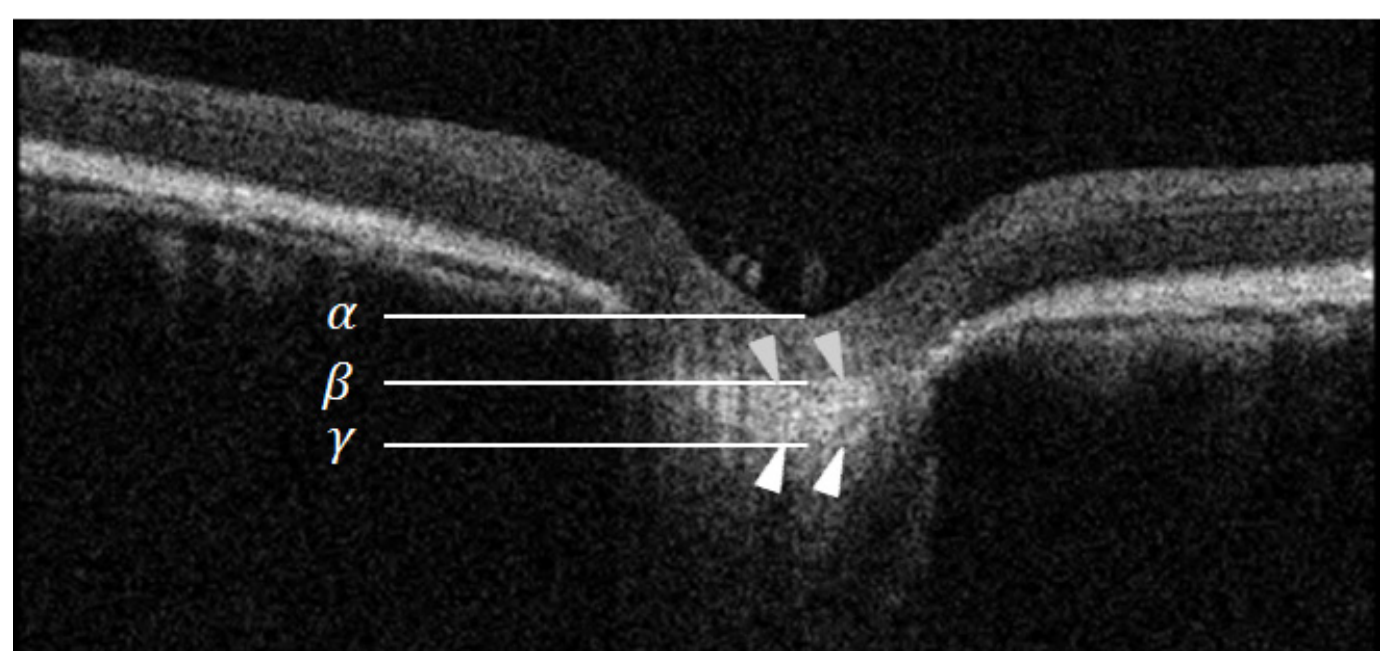

Fig. 2. Definition of thicknesses of the prelaminar tissue and LC. Line $\alpha$ is the optic cup surface, line $\beta$ is the anterior border of the LC, and line $\gamma$ is the posterior border of the LC. 


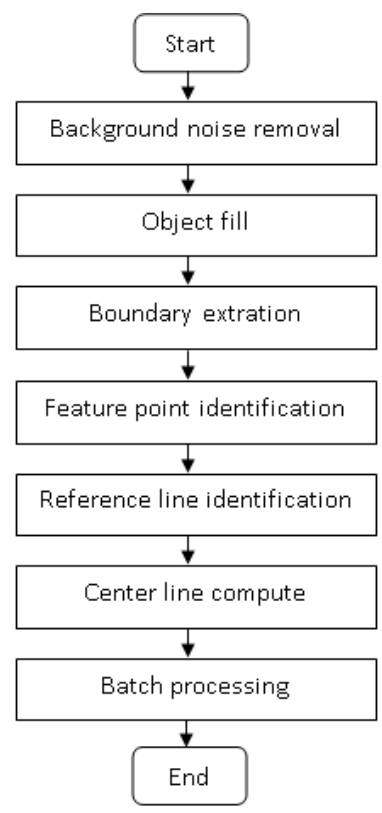

Fig. 3. Algorithm flowchart of the program used to optimize the obtained images for later thickness measurement.

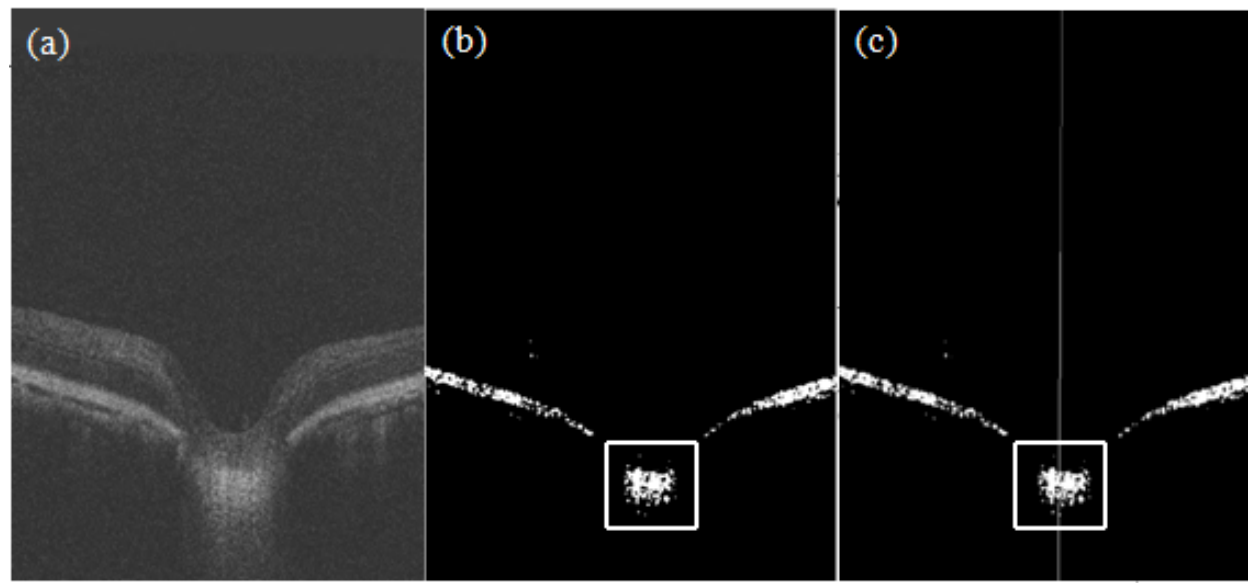

Fig. 4. Signal areas segmented with program.

\subsection{Calculation of the LC curvature}

The middle fifteen images covering the $\mathrm{ONH}$ were selected for curvature calculations from $128 \mathrm{~B}$ scan images, shown in Figure 5 from $-\mathrm{k}$ to $+\mathrm{k}$. From each piece of the SD-OCT images, whose matrix size is $512 \times 650$, we choose then points on the anterior LC surface to calculate the curvature. For example, point $\mathrm{A}$ in Figure 5 is one point. We could extract two-dimensional pixel coordinates of the points. Considering the area volume was $6.0 \times 2.3 \mathrm{~mm}^{2}$, the two-dimensional coordinates parallel to the $\mathrm{X}$ and $\mathrm{Y}$ axis can be obtained according to expression (1). The dimension of $\mathrm{Z}$ direction for $128 \mathrm{~B}-$ scan images is $6.0 \mathrm{~mm}$. Therefore, assuming that the two-dimensional coordinate of point A was (x,y), and the three-dimensional coordinate was ( $\left.\mathrm{x}^{\prime}, \mathrm{y}^{\prime}, \mathrm{z}^{\prime}\right)$, the relation between them was as follows: 

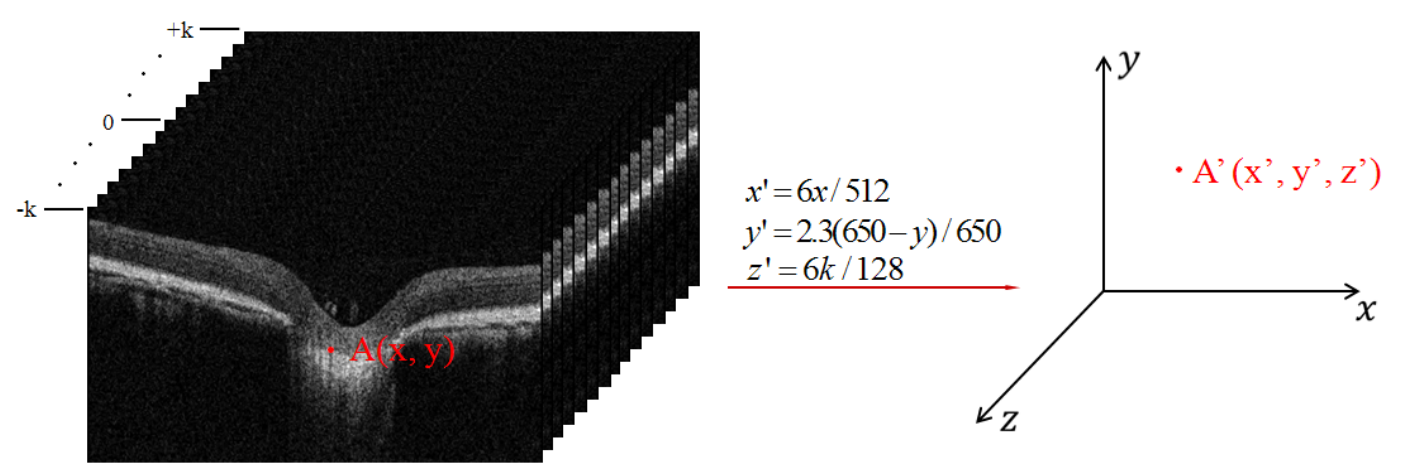

Fig. 5. The diagram of coordinate transformation in the study.

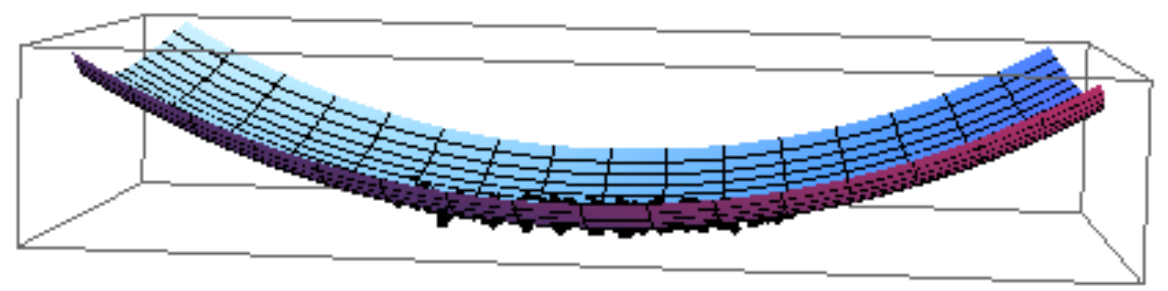

Fig. 6. An illustration of curvature calculation result.

$$
x^{\prime}=6 x / 512, y^{\prime}=2.3(650-y) / 650, z^{\prime}=6 k / 128,
$$

Where $k$ is the ordinal number from the middle piece, which ranged from -7 to +7 (Figure 5).

To calculate the curvature of the LC, we used Mathematica 8.0 (Wolfram Research, Champaign, USA) to fit the border surface and acquired the curvature radius. A spherical model was employed for the fitting work. Figure 6 is an illustration of the results.

\subsection{Statistical analysis}

All datasets are reported as mean $\pm \mathrm{SD}$. A $t$-test was performed using SPSS 17.0 (IBM, Armonk, NY, USA) with $P$ values $<0.05$ indicating statistical significance.

\section{Results}

The physiological IOPs of the experimental subjects (mean \pm SD: $23.4 \pm 2.77 \mathrm{~mm} \mathrm{Hg}$ ) were measured before the anterior chamber perfusion.

B-scan images used for measurement and analysis had high quality scores. We chose three middle $\mathrm{ONH}$ pictures to measure the thicknesses in each experimental treatment condition. The mean thick- 
ness of the LC and prelaminar tissue was $205 \pm 26 \mu \mathrm{m}$ (mean \pm SD) and $212 \pm 47 \mu \mathrm{m}$ (mean \pm SD), respectively.

The relationship between IOP and thickness measurements is presented in Table 1 and Table 2. In the acute high IOP model, the thickness of the prelaminar tissue and LC decreased with increases in IOP. Significant reduction $(\mathrm{P}<0.05)$ of the prelaminar tissue thickness was observed at $100 \mathrm{~mm} \mathrm{Hg}$ IOP pressure since the initial scanning under IOP native pressure. The thickness decline in the LC first reached statistical significance at an IOP of $60 \mathrm{~mm} \mathrm{Hg}$. The curve fitting of thicknesses in the acute high IOP model are shown in Figure 7. The linear expression is $T(p)=-7.8137 p+216.45$ $\left(R^{2}=0.9212\right)$ for prelaminar tissue thickness and $T(p)=-8.5507 p+210.69\left(R^{2}=0.9612\right)$ for LC thickness. In order to know the relationship between the thickness variation and IOP, curve fittings of the force-deformation diagram of the prelaminar tissue and LC (Figure 8) were performed. The fitting expression was $p=17.6868+22.5128 e^{0.0361 \Delta T} \quad\left(\quad R^{2}=0.7362\right) \quad$ and $p=3.8072+20.1388 e^{0.0455 \Delta T}\left(R^{2}=0.9972\right)$, respectively.

The thinning of the LC first reached statistical significance after the high IOP had been maintained for three hours. However, there was no significant difference in prelaminar tissue thinning. To understand the relationship between the deformations and the period where the IOP was maintained at 100 $\mathrm{mm} \mathrm{Hg}$, creep curves (Figure 9) were generated and logarithmic equations were adopted to fit the curves. For the description to be clearer, we normalized the stresses, -- the thickness of different duration minuses the original thickness and then divided by the difference between the thickness of 100 $\mathrm{mm} \mathrm{Hg} 0 \mathrm{~h}$ and thickness of the physiological condition. All the differences were absolute values. Assuming that the thickness of the original condition was $a$, the value of the $100 \mathrm{~mm} \mathrm{Hg} 0 \mathrm{~h}$ was $b$, and the value of the $100 \mathrm{~mm} \mathrm{Hg} 1 \mathrm{~h}$ (for example) was $c$, the normalization value $\mathrm{k}$ was as follows:

$$
\mathrm{k}=|\mathrm{c}-\mathrm{a}| /|\mathrm{b}-\mathrm{a}|
$$

so when $100 \mathrm{~mm} \mathrm{Hg} \mathrm{kept} \mathrm{for} 0 \mathrm{~h}$, the $k$ was 1 . The fitting expressions were as follows: $J(t)=1+0.67068\left(1-e^{-\frac{t}{0.62931}}\right)\left(R^{2}=0.8066\right)$, for the creep curve of the prelaminar tissue, and $J(t)=1+1.35163\left(1-e^{-\frac{t}{1.81194}}\right)\left(R^{2}=0.9517\right)$, for the creep curve of the LC.

The curvature of the LC in vivo was calculated by the method of surface fitting. Considering the shape of anterior LC surface, we adopted a spherical equation to fit the surface. In order to precisely calculate the curvature of the LC, extraction work of two-dimensional pixel coordinates was performed by two independent workers $(\mathrm{QZ}$ and another worker knowing nothing correlative about the data processing). The relationship between IOP and the LC curvature is exhibited in Tables 3 and 4 . The root mean square (RMS) was used to evaluate the correlation between the two calculations. RMS of the two calculations was 0.0838 in the acute high IOP model and 0.0679 in the static high IOP model. The result showed that the relationship between IOP and the LC curvature is reliable. 
Prelaminar tissue

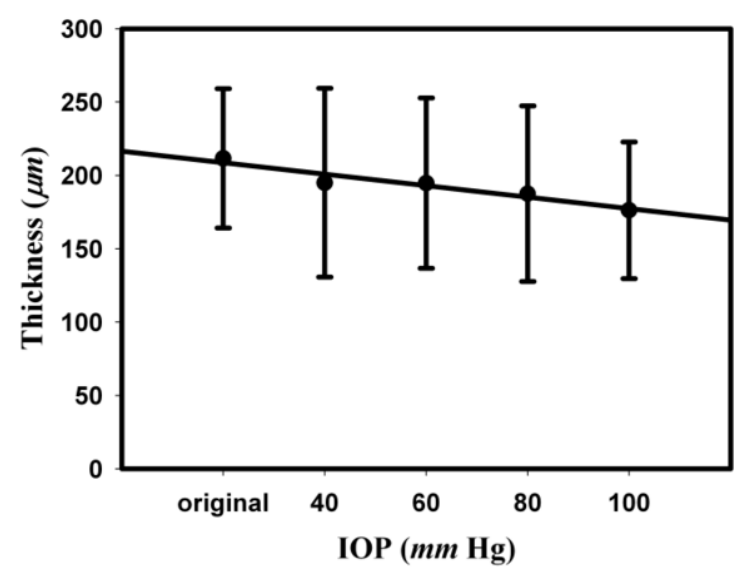

Lamina cribrosa

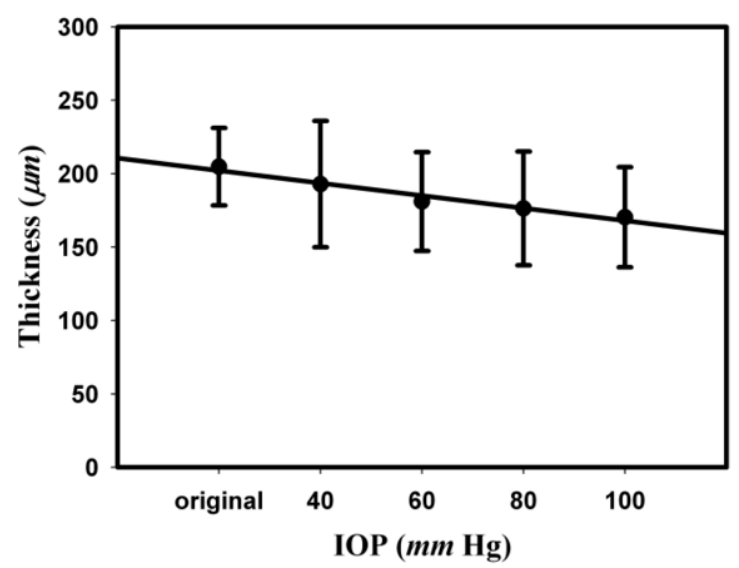

Fig. 7. Curve fitting the thickness of the prelaminar tissue and LC in acute high IOP model, $n=5$.

Prelaminar tissue

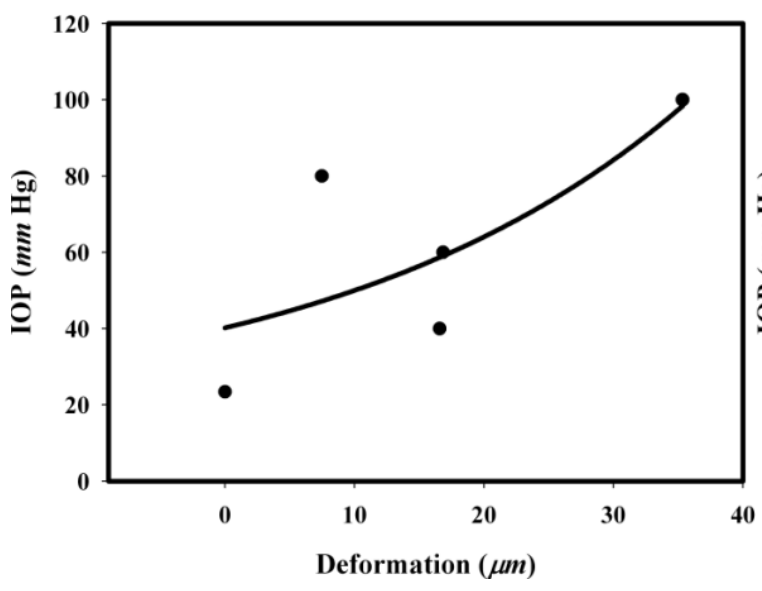

Lamina cribrosa

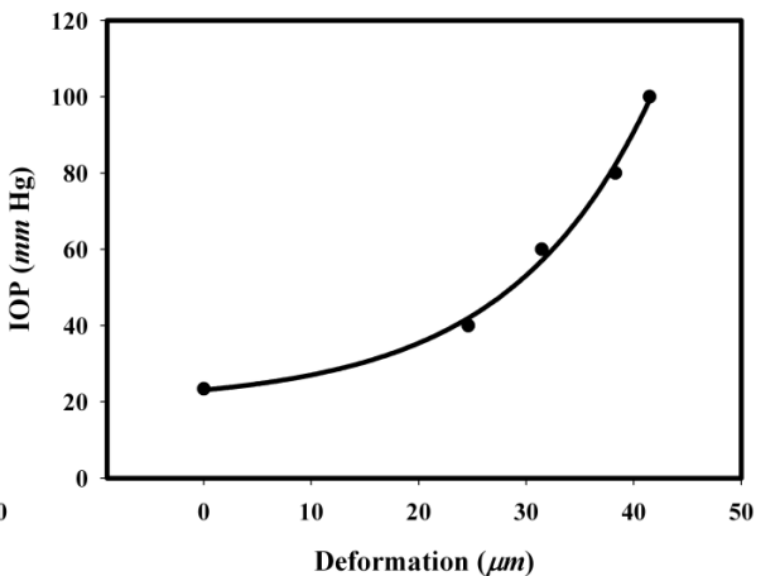

Fig. 8. The load-displacement curves.
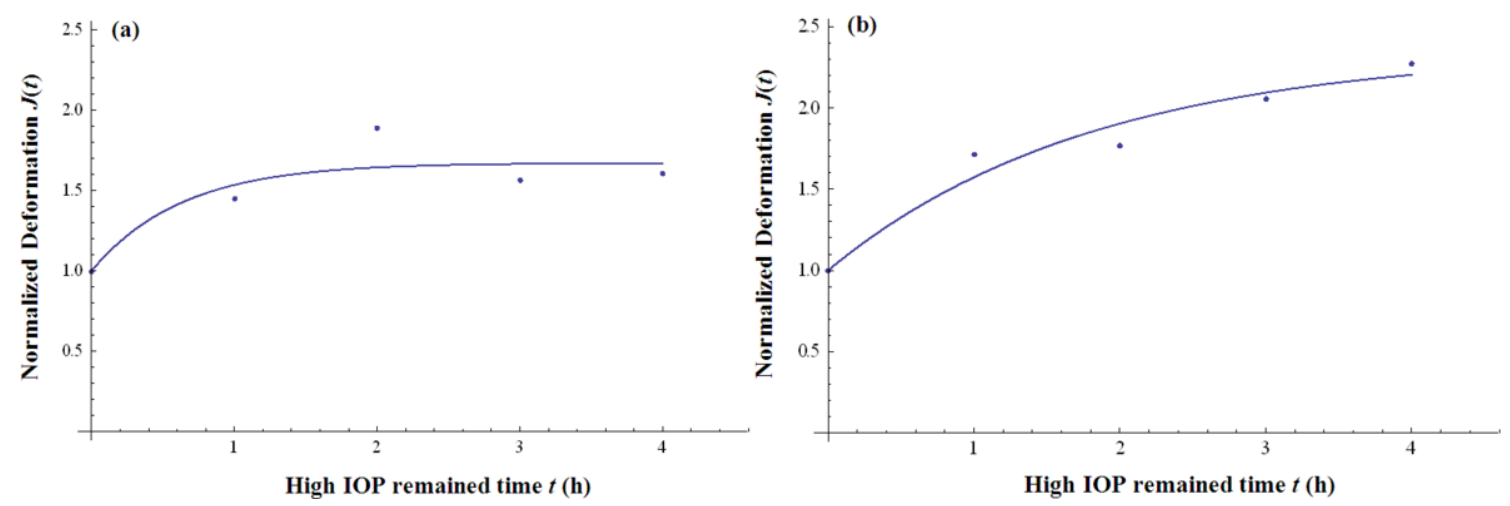

Fig. 9. The creep curve. (a) is of prelaminar tissue, (b) is of LC. 


\section{Discussion}

Mechanical compression of axons within LC has been suggested as a mechanism for damage in glaucoma. In vivo elevation of IOP may alter optic nerve tissue pressures, and consequently result in the deformation of the LC. In this study, we studied the changes in the LC shapes at different IOPs. Viscoelasticity is the property of materials that exhibit both viscous and elastic characteristics when undergoing deformation. Considering biological soft tissues are usually nonlinear, viscoelastic materials [32], we investigated the temporal impact on the deformation process of LC while IOP was conditioned at $100 \mathrm{~mm} \mathrm{Hg}$.

We choose cats as the experimental subjects because the LC of cats is well developed and the number of retinal ganglion cells it contains is fairly high [29,33,34]. We found the LC of cat eyes located more anteriorly within the ONH compared with humans, which may account for the differences found in clinical research.

In this study, we measured the thickness of the LC using 3D SD-OCT with an 840-nm light source. The vertical resolution was five $\mu \mathrm{m}$. Imaging of the LC was carried out using the choroidal mode, which has the equal functionality to the enhanced depth-imaging technique. Therefore, the location of the posterior laminar surface was clearly visible [25]. Although the mean cross-sectional area of the LC in normal cat eyes has been studied [31,33], the LC thickness measurements in cats has not been carried out in previous studies. We found the thickness of the LC thinned with increases in IOP. The thickness variation of the LC was correlated significantly with increases in IOP when the IOP was higher than $60 \mathrm{~mm} \mathrm{Hg}$. Our results are consistent with the results from Fatehee [27] and Jonas [35]. There are several studies that reported compression of the lamina occurs in the early, moderate, and severe stages of glaucomatous damage $[9,12,16,17,26]$. The advantage of our study is the determination of the thicknesses of the LC at five different IOPs in the same sample. Therefore, we obtained thickness variations of the LC with IOP by curve fitting. The results showed that exponential function fitting was better than linear function fitting, which was consistent with the nonlinear loaddisplacement property found in soft tissues.

When an acute glaucoma attacks, high IOP may keep harming the eye tissues for several hours. We measured the thickness variation of the LC while the IOP was maintained at $100 \mathrm{~mm} \mathrm{Hg}$ for 4 hours. The results demonstrated the thickness of the LC reduces as the duration of high IOP sustains, which shows the viscoelasticity of the LC [36].

The prelaminar layer of the $\mathrm{ONH}$ is predominantly neural in composition. We found that the thickness of the prelaminar tissue decreased with increases in IOP, and the thickness variation compared with the original value had statistically significant difference when IOP=100 $\mathrm{mm} \mathrm{Hg}$. The relationship between the thickness variation of the LC and IOP can also be modeled by an exponential function. We also measured the thickness variation of the prelaminar tissue during IOP at $100 \mathrm{~mm} \mathrm{Hg}$ for 4 hours. The data suggested that the thickness of the prelaminar tissue of the LC had no obvious relationship with the duration time of high IOP and the coefficient of determination for the fitting curve was 0.8066 . Considering the mechanical properties of soft tissue, it is more difficult to deform with an increase of pressure at a region that already has high stress. Likewise, it is possible that when IOP reaches $100 \mathrm{~mm} \mathrm{Hg}$, the prelaminar tissue has already reached the region of high stress. This could explain why deformation of the prelaminar of the LC was small when IOP was kept at $100 \mathrm{~mm} \mathrm{Hg}$.

The posterior displacement of the LC has attracted great academic attention $[10,22,37,38]$. The results of a previous in vitro study showed that elevated IOP can cause the LC to deflect posteriorly [37] and lead to changes in LC curvature [39]. Agoumi et al. [38] visualized the change of the ONH structure in human eyes after acute IOP elevation using SD-OCT in vivo. They observed a significant pre- 
Table 1

Thickness of the prelaminar tissue and the LC in the acute high pressure model $(\mathrm{n}=5)$

\begin{tabular}{llllll}
\hline & original & 40 & 60 & 80 & 100 \\
\hline PTT & $212 \pm 47$ & $195 \pm 64$ & $195 \pm 58$ & $188 \pm 60$ & $176 \pm 47^{*}$ \\
\hline LCT & $205 \pm 26$ & $193 \pm 43$ & $181 \pm 33^{*}$ & $177 \pm 38^{*}$ & $172 \pm 32^{*}$ \\
\hline
\end{tabular}

Note: * stands for the thickness compared with the original value has statistical significance $(\mathrm{P}=0.0491,0.0425,0.0294,0.0045)$. All data measured in micrometer and compared with the original condition.

Table 2

Thickness of the prelaminar tissue and the LC in the static high IOP model $(\mathrm{n}=5)$

\begin{tabular}{|c|c|c|c|c|c|}
\hline & $0 \mathrm{~h}$ & $1 \mathrm{~h}$ & $2 \mathrm{~h}$ & $3 \mathrm{~h}$ & $4 \mathrm{~h}$ \\
\hline PTT & $176 \pm 47$ & $184 \pm 54$ & $168 \pm 43$ & $170 \pm 36$ & $167 \pm 29$ \\
\hline LCT & $172 \pm 32$ & $158 \pm 19$ & $153 \pm 20$ & $146 \pm 15^{*}$ & $141 \pm 19 *$ \\
\hline
\end{tabular}

Table 3

Curvature of the LC changes in acute model $(n=5)$

\begin{tabular}{llllll}
\hline & original & 40 & 60 & 80 & 100 \\
\hline Curvature & 0.48293 & 0.48610 & 0.39782 & 0.38062 & 0.39096 \\
\hline \multicolumn{7}{r}{ Note: The change of curvature has no statistical significance. }
\end{tabular}

Table 4

Curvature of the LC changes in remained model $(n=5)$

\begin{tabular}{llllll}
\hline & $0 \mathrm{~h}$ & $1 \mathrm{~h}$ & $2 \mathrm{~h}$ & $3 \mathrm{~h}$ & $4 \mathrm{~h}$ \\
\hline Curvature & 0.39096 & 0.36119 & 0.36461 & 0.31078 & 0.36653 \\
\hline & Note: The change of curvature has no statistical significance.
\end{tabular}

laminar tissue displacement with no significant posterior displacement of the LC. Similarly, we did not observe noticeable posterior displacement of the LC.

In conclusion, using EDI SD-OCT we were able to visualize the LC and study the shape changes of the LC. The measurement of the thickness alteration of the LC and prelaminar tissue under different IOP was conducted. Our results demonstrated that the thickness of both the prelaminar tissue and LC thinned as the intraocular pressure was elevated. The thicknesses also decreased as the IOP was maintained at $100 \mathrm{~mm} \mathrm{Hg}$ for 4 hours.

\section{Acknowledgement}

The work was funded by the National Natural Science Foundation of China (Nos. 31070840, Nos. 11102123), and the Natural Science Foundation of Beijing (Nos. 3122010, 7142024), and also by the funding project for Academic Human Resources Development in Institutions of Higher Learning Under the Jurisdiction of Beijing Municipality (No. PHR201110506). This work was also supported by the Open Project Program of Beijing Key Laboratory of Fundamental Research on Biomechanics in Clinical Application, China. 


\section{References}

[1] S. Thanosand and R. Naskar, Correlation between retinal ganglion cell death and chronically developing inherited glaucoma in a new rat mutant, Experimental Eye Research 79 (2004), 119-129.

[2] N. Sredar, K.M. Ivers, H.M. Queener, G. Zouridakis and J. Porter, 3D modeling to characterize lamina cribrosa surface and pore geometries using in vivo images from normal and glaucomatous eyes, Biomedical Optics Express 4 (2013), $1153-1165$

[3] R.N. Weinreb and P.T. Khaw, Primary open-angle glaucoma, The Lancet 363 (2004), 1711-1720.

[4] N. Soman and R. Banerjee, Artificial vitreous replacements, Bio-Medical Materials and Engineering 13 (2003), 59-74.

[5] D.S. Minckler, A.H. Bunt and G.W. Johanson, Orthograde and retrograde axoplasmic transport during acute ocular hypertension in the monkey, Investigative Ophthalmology \& Visual Science 16 (1977), 426-441.

[6] H.A. Quigley, E.M. Addicks, W.R. Green and A.E. Maumenee, Optic nerve damage in human glaucoma, II, The site of injury and susceptibility to damage, Archives of Ophthalmology 99 (1981), 635-649.

[7] H.A. Quigley and W.R. Green, The histology of human glaucoma cupping and optic nerve damage: Clinicopathologic correlation in 21 eyes, Ophthalmology 86 (1979), 1803-1830.

[8] A.J. Bellezza, C.J. Rintalan, H.W. Thompson, J.C. Downs, R.T. Hart and C.F. Burgoyne, Deformation of the lamina cribrosa and anterior scleral canal wall in early experimental glaucoma, Investigative Ophthalmology \& Visual Science 44 (2003), 623-637.

[9] H.Y. Park, S.H. Jeon and C.K. Park, Enhanced depth imaging detects lamina cribrosa thickness differences in normal tension glaucoma and primary open-Angle glaucoma, Ophthalmology 119 (2012), 10-20.

[10] H.A. Quigley and E.M. Addicks, Regional differences in the stucture of the laminal cribeosa and their relation to glaucomatous optic nerve damage, Archives of Ophthalmology 99 (1981), 137-143.

[11] H.A. Quigley, R.M. Hohman, E.M. Addicks, R.W. Massof and W.R. Green, Morphologic changes in the lamina cribrosa correlated with neural loss in open-angle glaucoma, American Journal of Ophthalmology 95 (1983), 673-691.

[12] R. Ren, N. Wang, B. Li, L. Li, F. Gao, X. Xu and J.B. Jonas, Lamina cribrosa and peripapillary sclera histomorphometry in normal and advanced glaucomatous chinese eyes with various axial length, Investigative Ophthalmology \& Visual Science 50 (2009), 2175-2184.

[13] H.A. Quigley and E.M. Addicks, Chronic experimental glaucoma in primates-II. Effect of extended intraocular pressure elevation on optic nerve head and axonal transport, Investigative Ophthalmology \& Visual Science 19 (1980), 137-152.

[14] I.A. Sigal, J.G. Flanagan, I. Tertinegg and C.R. Ethier, Reconstruction of humanoptic nerve heads for finite element modeling, Technology and Health Care 13 (2005), 313-329.

[15] L. Kagemann, H. Ishikawa, G. Wollstein, P.M. Brennen, K.A. Townsend, M.L. Gabriele and J.S. Schuman, Ultrahighresolution spectral domain optical coherence tomography imaging of the lamina cribrosa, Ophthalmic Surgery, Lasers \& Imaging 39 (2008), S126-S131.

[16] R. Inoue, M. Hangai, Y. Kotera, H. Nakanishi, S. Mori, S. Morishita and N. Yoshimura, Three-dimensional high-speed optical coherence tomography imaging of laminacribrosa in glaucoma, Ophthalmology 116 (2009), 214-222.

[17] E.J. Lee, T.W. Kim, R.N. Weinreb, K.H. Park, S.H. Kim and D.M. Kim, Visualization of the lamina cribrosa using enhanced depth imaging spectral-domain optical conerence tomography, American Journal of Ophthalmology 152 (2011), 87-95.

[18] E.J. Lee, T.W. Kim, R.N. Weinreb, M.H. Suh and H. Kim, Lamina cribrosa thickness is not correlated with central corneal thickness or axial length in healthy eyes: Central corneal thickness, axial length, and lamina cribrosa thickness, Graefes Archive for Clinical and Experimental Ophthalmology 251 (2013), 847-854.

[19] J.M. Mari, N.G. Strouthidis, S.C. Park and M.J. Girard, Enhancement of lamina cribrosa visibility in optical coherence tomography images using adaptive compensation, Investigative Ophthalmology \& Visual Science 54 (2013), 22382247.

[20] E.J. Lee, T.W. Kim, R.N. Weinreb, M.H. Sub, M. Kang, K.H. Park, S.H. Kim and D.M. Kim, Three-dimensional evaluation of the lamina cribrosa using spectral-domain optical coherence tomography in glaucoma, Investigative Ophthalmology \& Visual Science 53 (2012), 198-204.

[21] E.J. Lee, T.W. Kim, R.N. Weinreb and H. Kim, Reversal of lamina cribrosa displacement after intraocular pressure reduction in open-angle glaucoma, Ophthalmology 120 (2013), 553-559.

[22] E.J. Lee, T.W. Kim and R.N. Weinreb, Reversal of lamina cribrosa displacement and thickness after trabeculectomy in glaucoma, Ophthalmology 119 (2012), 1359-1366.

[23] E.J. Lee, T.W. Kim and R.N. Weinreb, Improved reproducibility in measuring the laminar thickness on enhanced depth imaging SD-OCT images using maximum intensity projection, Investigative Ophthalmology \& Visual Science $\mathbf{5 3}$ (2012), 7576-7582. 
[24] E.J. Lee, T.W. Kim and R.N. Weinreb, Variation of lamina cribrosa depth following trabeculectomy, Investigative Ophthalmology \& Visual Science 54 (2013), 5392-5399.

[25] A. Miki, Y. Ikuno, Y. Jo and K. Nishida, Comparison of enhanced depth imaging and high-penetration optical coherence tomography for imaging deep optic nerve head and parapapillary structures, Clinical Ophthalmology 7 (2013), 1995-2001.

[26] H.Y. Park and C.K. Park, Diagnostic capability of lamina cribrosa thickness by enhanced depth imaging and factors affecting thickness in patients with glaucoma, Ophthalmology 120 (2013), 745-752.

[27] N. Fatehee, P.K. Yu, W.H. Morgan, S.J. Cringle and D.Y. Yu, The impact of acutely elevated intraocular pressure on the porcine optic nerve head, Investigative Ophthalmology \& Visual Science 52 (2011), 6192-6198.

[28] N.G. Strouthidis, B. Fortune, H. Yang, I.A. Sigal and C.F. Burgoyne, Effect of acute intraocular pressure elevation on the monkey optic nerve head as detected by spectral domain optical coherence tomography, Investigative Ophthalmology \& Visual Science 52 (2011), 9431-9437.

[29] M.C. Albrecht, Comparative anatomy of the optic nerve head and inner retina in non-primate animal models used for glaucoma research, The Open Ophthalmology Journal 2 (2008), 94-101.

[30] J. Qiu, X. Qian, Q. Cui, C. Mu, K. Zhang, B. Na, H. Song, H. Quan and Z. Liu, Three-dimensional reconstruction and finite element modeling analysis of the rabbit optic nerve head in acute high intraocular pressure, Japanese Journal of Applied Physics 51(2012), 067001-1-067001-6.

[31] J. Lai and Q. Wei, Automatic lung fields segmentation in CT scans using morphological operation and anatomical information, Bio-Medical Materials and Engineering 24 (2014), 335-340.

[32] Y.C. Fung, Biomechanics: Mechanical Properties of Living Tissues, 2nd edi., Springer-Verlag New York Inc., New York, 1993.

[33] R.L. Radius and B. Bade, The anatomy at the lamina cribrosa in the normal cat eye, Archives of Ophthalmology 100 (1982), 1658-1660.

[34] R.L. Radius and B. Bade, Axonal transport interruption and anatomy at the lamina cribrosa, Archives of Ophthalmology 100 (1982), 1661-1664.

[35] J.B. Jonas, S.S. Hayreh and T. Yong, Thickness of the lamina cribrosa and peripapillary sclerain Rhesus monkeys with onglaucomatous or glaucomatous optic neuropathy, Acta Ophthalmologica 89 (2011), e423-e427.

[36] A.J. Bellezza, R.T. Hart and C.F. Burgoyne, The optic nerve head as a biomechanical structure: Initial finite element modeling, Investigative Ophthalmology \& Visual Science 41 (2000), 2991-3000.

[37] D.B. Yan, F.M. Coloma, A. Metheetrairut, G.E. Trope, J.G. Heathcote and C.R. Ethier, Deformation of the lamina cribrosa by elevated intraocular pressure, British Journal of Ophthalmology 78 (1994), 643-648.

[38] Y. Agoumi, G.P. Sharpe, D.M. Hutchison, M.T. Nicolela, P.H. Artes and B.C. Chauhan, Laminar and prelaminar tissue displacement during intraocular pressure elevation in glaucoma patients and healthy controls, Ophthalmology 118 (2011), 52-59.

[39] H. Yang, J.C. Downs, C. Girkin, L. Sakata, A. Bellezza, H. Thompson and C.F. Burgoyne, 3-D histomorphometry of the normal and early glaucomatous monkey optic nerve head: lamina cribrosa and peripapillary scleral position and thickness, Investigative Ophthalmology \& Visual Science 48 (2007), 4597-4607. 